\title{
ANTI-PLANE SHEAR WAVES IN A FIBRE-REINFORCED COMPOSITE WITH A NON-LINEAR IMPERFECT INTERFACE
}

\author{
Vladyslav V. Danishevs'kyy', Julius D. Kaplunov², Graham A. Rogerson ${ }^{2}$
}

${ }^{1}$ Department of Structural Mechanics and Strength of Materials, Prydniprovska State Academy of Civil Engineering and Architecture, Ukraine

${ }^{2}$ School of Computing and Mathematics, Keele University, UK

\begin{abstract}
The propagation of nonlinear elastic anti-plane shear waves in a unidirectional fibre-reinforced composite material is studied. A model of structural nonlinearity is considered, for which the nonlinear behaviour of the composite solid is caused by imperfect bonding at the "fibre-matrix" interface. A macroscopic wave equation accounting for the effects of nonlinearity and dispersion is derived using the higher-order asymptotic homogenization method. Explicit analytical solutions for stationary nonlinear strain waves are obtained. This type of nonlinearity has a crucial influence on the wave propagation mode: for soft nonlinearity, localised shock (kink) waves are developed, while for hard nonlinearity localised bell-shaped waves appear. Numerical results are presented and the areas of practical applicability of linear and nonlinear, long- and shortwave approaches discussed.
\end{abstract}

Key words: nonlinear wave; solitary wave; composite material; imperfect bonding; asymptotic homogenization.

1. Introduction. Elastic waves propagating in heterogeneous solids can undergo the effects of nonlinearity and dispersion. Nonlinearity may arise through geometrical, physical or structural mechanisms (e.g., Lur'e, 1990). We study a problem for which the nonlinear behaviour of a composite is associated with 
imperfect bonding conditions at the interface between constitutive components. This is an example of structural nonlinearity, with the nonlinearity directly related to the presence of a microstructure. Dispersion on the other hand can be classified as geometrical or structural. Geometrical dispersion is typical for wave-guides and finite-size bodies (e.g., waves in beams and plates). Structural dispersion may be caused by the heterogeneity of a composite solid, with successive reflections and refractions of local waves at the matrix-inclusion interfaces leading to scattering of the overall wave field.

Nonlinearity induces a pumping of energy form the low- to the high-frequency part of the spectrum, with higher-order modes generated and continuous localization of energy occurring, making the wave front steeper. In contrast, dispersion provides scattering of energy and decreases the slope of the wave front. When nonlinearity and dispersion act together, they may balance the influence of each other (Kunin, 1982). In such a case, stationary nonlinear waves of permanent shape and velocity can propagate. Nonlinear strain waves play an important role in the mechanical behaviour of composite materials and structures. An increase in nonlinearity leads to the formation of localized solitary waves. This process is accompanied by essential strain amplitude growth, possibly resulting in the development of local plastic zones and/or cracks. Therefore, nonlinear dynamic effects can become a crucial factor affecting strength and durability of engineering structures.

In many cases, nonlinear elastic moduli of heterogeneous solids are very sensitive to the properties of microstructure (see, for example, Zaitsev et al. (2006). Measuring the characteristics of nonlinear waves enables detection of very small variations of the internal texture of the medium at a level not possible within a linear framework (Zumpano and Meo, 2008; Polimeno and Meo, 2008). This provides the possibility of developing new, more precise, methods of the acoustic diagnostic and non-destructive testing in engineering, geophysics, biomechanics and other areas dealing with heterogeneous materials and structures. 
The propagation of nonlinear strain waves in elastic solids has been intensively studied. For a comprehensive review of the subject we refer to the books by Jeffrey and Engelbrecht (1994), Maugin (1999), Samsonov (2001), Erofeev (2003), and Porubov (2003). Many authors considered homogeneous systems, with dispersive properties mainly determined by geometrical factors. At the same time, the effect of structural dispersion, related to the scattering of nonlinear waves by the microstructure, were not studied in great detail.

The influence of the microstructure can be modelled by allowing the elastic medium additional internal degrees of freedom, an idea originally proposed over 100 years ago by Cosserat (1909) and Le Roux (1911). Following Cosserat theory, a number of higher-order continuum models were developed by Mindlin (1964), Sun et al. (1968), Herrmann and Achenbach (1968). More recently, similar approaches were adopted to describe the propagation of nonlinear strain waves in miscrostructured solids (Engelbrecht et al., 2007, 2011; Porubov et al., 2004, 2009; Randrüüt and Braun, 2010). A recent review of the modelling of heterogeneous media in terms of internal variables was presented by Berezovski et al. (2011a, b).

From a mathematical viewpoint, the aforementioned approaches supplement the constitutive equations of motion with some additional higher-order gradient terms accounting for the effects of dispersion. The coefficients at the dispersive terms represent what might be thought of as phenomenological parameters. In some cases these may be determined experimentally; however, for most industrial materials their magnitudes remain unknown. An alternative way to predict the influence of microstructures is provided by the asymptotic homogenization method (AHM). According to this approach, physical fields in a spatially periodic heterogeneous medium are represented by a two-scale asymptotic expansion in powers of a small parameter $\eta=l / L$, where $l$ is the size of the unit cell and $L$ is the typical wavelength. This leads to a decomposition of the final solution into global and local components; the latter are evaluated from a recurrent sequence of cell boundary value problems (BVPs). Application of the volume-integral 
homogenizing operator allows us to obtain a homogenized constitutive equation that describes the macroscopic behaviour of the medium. It is important to note that the coefficients of the homogenized equation (so-called effective moduli) are evaluated based on information about the properties of the components and the geometry of the microstructure. Thus, in contrast to Cosserat-type approaches, the homogenized model incorporates data about the internal composition of the material.

From its conception, the AHM was intended for the determination of quasistatic properties of heterogeneous media and structures (e.g., Bensoussan et al. (1978), Sanchez-Palencia (1980), Bakhvalov and Panasenko (1989); Kalamkarov et al. (2009)). Taking into account higher-order terms, with respect to $\eta$, extended the area of applicability of the homogenized models and provided a mechanism to predict the effect of structural dispersion (Boutin and Auriault, 1993; Chen and Fish, 2001; Fish and Chen, 2004; Andrianov et al., 2008; Soubestre and Boutin, 2012; Auriault and Boutin, 2012). Non-local effects resulting both from highanisotropy and high-contrast of composite structures were studied by Cherednichenko et al. (2006), Smyshlyaev (2009), Soubestre and Boutin (2012). It should be noted that macroscopic dynamic equations obtained by the AHM are valid only in the long-wave case, when $l \ll L$. Recently, Craster et al. (2014) have shown a subtle analogue between the long-wave asymptotic procedures underlying approximate formulations for periodic media and for functionally graded waveguides. The conventional AHM seems to be a counterpart of the classical theories for thin plates, shells and rods. A theoretical framework for the asymptotic theories of long wave motion in plates and layered media was developed by Rogerson et al. (2006, 2007, 2009), Lutianov and Rogerson (2010), Mukhomodyarov and Rogerson (2012). Homogenization of nonlinear dynamic problems was considered by Andrianov et al. $(2011,2013)$.

When the wavelength of a travelling signal decreases and becomes comparable to the size of the microstructure, a heterogeneous elastic solid exhibits a 
complicated sequence of pass and stop frequency bands. In the literature, they are also referred to as phononic bands (by analogy with the photonic bands arising for electromagnetic and optical waves in heterogeneous dielectric media). Thus, the composite plays the role of a discrete wave filter. If the frequency falls within a stop band, a stationary wave is excited and neighbouring heterogeneities (e.g. particles) vibrate in alternate directions. At a macrolevel, the amplitude of the global wave attenuates exponentially, so no propagation is possible. Phononic bands can be theoretically predicted using the Floquet-Bloch approach (Bloch, 1928). This approach has been documented in the book by Brillouin (2003, 2nd edn.) and utilized by many authors (see, for example, Movchan et al. (2002, 2006, 2007), McPhedran et al. (2009) and references therein).

Craster et al. (2010a, 2010b, 2012) and Nolde et al. (2011) proposed a generalization of the AHM making it suitable for the analysis of high-frequency waves. The key point was to choose a zero-order approximation, not at the quasistatic limit $(\eta \rightarrow 0)$, but at the edges of the high-frequency phononic bands.

In this present paper, we apply the AHM to the modelling of anti-plane shear waves propagating in a fibre-reinforced composite material with imperfect interface bonding between the matrix and fibres. For engineering materials, the properties of the interface may be subjected to various factors, such as the presence of thin coating layers, chemical reactions or mechanical damages. From the mathematical viewpoint, the effect of imperfect bonding can be predicted by assuming that the displacement jump across the interface is related to the interfacial stress by a certain cohesion function. This approach is general and can describe different types of interfaces independently of the physical reasons of the debonding.

In the simplest case, the cohesion function is assumed linear, the interface then acting like an elastic spring. The spring-type interface model was originally proposed by Goland and Reissner (1944). In the theory of composites, it was first introduced by Mal and Bose (1975) and later employed by a number of authors. 
Variational formulations for the imperfect bonding conditions were presented by Hashin (1992), Lipton and Vernescu (1995). Limiting cases of very soft and very stiff interfaces were analysed by Benveniste and Miloh (2001). Needleman (1990, 1992), Tvergaard (1990, 1995), Espinosa et al. (1998, 2000) considered more sophisticated cohesion functions and simulated various scenarios of the debonding process. While reducing the cohesion, the stress field (supported by the interface) increases in magnitude, achieves a maximum, and ultimately falls to zero when complete separation occurs. It is therefore possible to track the evolution of the debonding process from its initial onset to complete separation and subsequent formation of voids. Nonlinear interfaces were considered by Levy and Dong (1988), Levy (2000), Nguyen and Levy (2009). An asymptotic simulation of the imperfect bonding was presented by Andrianov et al. $(2007,2010)$.

It should be noted that most of the interface models include a number of phenomenological parameters: the maximal interfacial traction, characteristic lengths of the interfacial displacements, interface shear-to-normal strength ratio, etc. Such quantities can not usually be identified a priori. At the same time, due to evident experimental difficulties. there is still very little effort to measure cohesive laws in real materials. As a successful example, we refer to Tan et al. (2005) who developed an experimental approach to determine the microscopic cohesive law in composite high explosives.

In our present contribution we specifically study a weakly nonlinear interface, with a cohesion function represented by a power series expansion in terms of nondimensional displacement jumps. The paper is organized as follows. In Section 2, an asymptotic model of the imperfect bonding is proposed and the input BVP introduced. In Section 3, the higher-order asymptotic homogenization procedure is developed and the macroscopic nonlinear wave equation obtained. In Section 4, the analytical solution for stationary nonlinear strain waves is derived in terms of elliptic functions. The analysis of the obtained results and numerical examples are presented in Section 5. Section 6 is devoted to the conclusions. 


\section{Asymptotic model of the imperfect bonding and input BVP problem.}

Let us consider a unidirectional fibre-reinforced composite consisting of an infinite matrix $\Omega^{(1)}$ and a periodic square array of cylindrical inclusions $\Omega^{(2)}$, see Fig. 1. It is supposed that geometrical and physical nonlinearity can be neglected, with the nonlinear behaviour of the composite caused by imperfect bonding at the matrixfibres interface $\partial \Omega$.

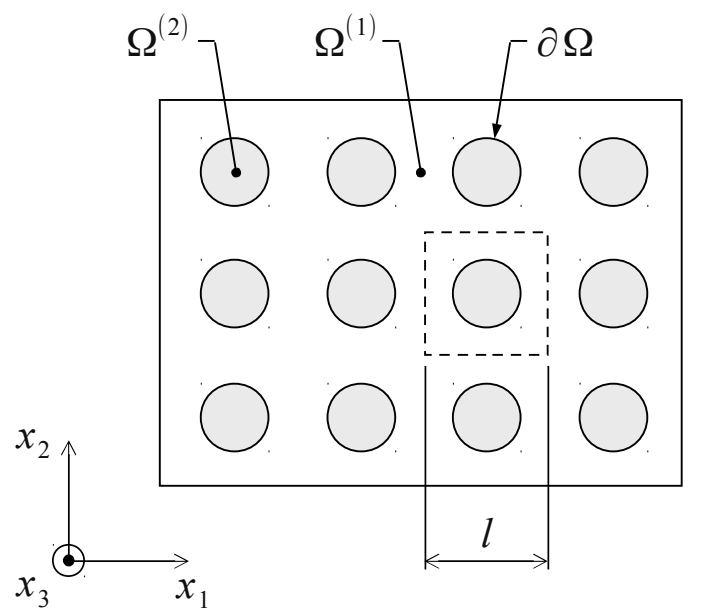

Fig 1. Fibre-reinforce composite under consideration.

We study elastic waves propagating in the plane $x_{1} x_{2}$. The parameters of the stress-strain state depend only on two spatial coordinates $x_{1}, x_{2}$. Generally, three type of waves can be realised: (i) in-plane longitudinal tensile/compression wave; (ii) in-plane transverse shear wave; and (iii) anti-plane transverse shear wave. The in-plane waves are coupled, whilst the anti-plane wave is uncoupled and, therefore, can be considered separately.

In this paper, we study anti-plane shear waves. The deformations occur under tangential stresses $\sigma_{13}, \sigma_{23}$. Normal stresses $\sigma_{11}, \sigma_{22}, \sigma_{33}$ as well as the tangential stress $\sigma_{12}$ are zero. The governing wave equation is as follows:

$$
\mu^{(n)} \nabla_{x}^{2} u^{(n)}=\rho^{(n)} \frac{\partial^{2} u^{(n)}}{\partial t^{2}}
$$


where $\mu^{(n)}$ is the shear modulus; $\rho^{(n)}$ is the density; $u^{(n)}$ is the displacement in the $x_{3}$ direction; $\nabla_{x}=\left(\partial / \partial x_{1}\right) \mathbf{e}_{1}+\left(\partial / \partial x_{2}\right) \mathbf{e}_{2} ; \mathbf{e}_{1}, \mathbf{e}_{2}$ are the Cartesian unit vectors. Here, and throughout this paper, the upper index $(n)$ refers to different components of the composite structure, $n=1,2$.

Let us consider the case of imperfect bonding at the interface $\partial \Omega$. The equilibrium state implies the equality of tangential stresses, thus:

$$
\sigma^{*}=\sigma^{(1)}=\sigma^{(2)} \text { at } \partial \Omega,
$$

where $\sigma^{(n)}=\mu^{(n)}\left(\partial u^{(n)} / \partial \mathbf{n}\right) ; \partial / \partial \mathbf{n}$ is the normal derivative to $\partial \Omega$. Weakening the bonding between the matrix and fibres leads to a jump in the displacement field across the interface. We suppose that the displacement jump $\Delta u^{*}=u^{(1)}-u^{(2)}$ is related to the interfacial stress $\sigma^{*}$ as follows:

$$
\sigma^{*}=f\left(\Delta u^{*}\right) \text { at } \partial \Omega
$$

where $f\left(\Delta u^{*}\right)$ is the so-called cohesion function. A phenomenological framework for describing the process of interfacial decohesion was presented by Needleman (1990, 1992) and Tvergaard (1990, 1995). Different types of cohesion functions were considered by Espinosa et al. (1998, 2000).

If the interface exhibits weakly nonlinear behaviour, the cohesion function can be assumed in the following form:

$$
\sigma^{*}=\mu_{1}^{*} \frac{\Delta u^{*}}{h}+\mu_{3}^{*}\left(\frac{\Delta u^{*}}{h}\right)^{3} \text { at } \partial \Omega
$$

where $h$ is the thickness of the interface. From the mathematical point of view, 
expression (4) can be considered as the first terms of Taylor series expansion of $f\left(\Delta u^{*}\right)$ in powers of $\Delta u^{*} / h$. The shear deformation is always symmetric, therefore, in the problem under consideration the cohesion function is odd, $f\left(\Delta u^{*}\right)=-f\left(-\Delta u^{*}\right)$, and expansion (4) includes only terms of odd powers. The coefficients $\mu_{1}^{*}, \mu_{3}^{*}$ can be interpreted, respectively, as the linear and the nonlinear shear modulus of the interface. Inversion of series (4) yields:

$$
\frac{\Delta u^{*}}{h}=\frac{\sigma^{*}}{\mu_{1}^{*}}-\frac{\mu_{3}^{*}}{\mu_{1}^{*}}\left(\frac{\sigma^{*}}{\mu_{1}^{*}}\right)^{3}+O\left[\left(\frac{\sigma^{*}}{\mu_{1}^{*}}\right)^{5}\right] \text { at } \partial \Omega .
$$

Let us introduce non-dimensional bonding parameters:

$$
\alpha=\frac{h \mu^{(1)}}{l \mu_{1}^{*}}, \quad \beta=\frac{\mu_{3}^{*}}{\mu_{1}^{*}}\left(\frac{\mu^{(1)}}{\mu_{1}^{*}}\right)^{2},
$$

where the thickness $h$ of the interface is normalised with respect to the size $l$ of the unit cell, and the elastic constants $\mu_{1}^{*}, \mu_{3}^{*}$ are normalised with the shear modulus $\mu^{(1)}$ of the matrix. Letting $h \rightarrow 0, \mu_{1}^{*} \rightarrow 0, \mu_{3}^{*} \rightarrow 0$, in the asymptotic limit it becomes possible to describe different rates of debonding depending on the magnitudes of the parameters $\alpha$ and $\beta$. Then, expression (5) reads

$$
u^{(1)}-u^{(2)}=\alpha l \frac{\sigma^{*}}{\mu^{(1)}}-\alpha \beta l\left(\frac{\sigma^{*}}{\mu^{(1)}}\right)^{3} \text { at } \partial \Omega .
$$

The case $\alpha=0$ corresponds to perfect bonding, $\alpha \rightarrow \infty$ - to complete separation of the components. At $\beta=0$ the interface is purely linear, whilst any increase in $|\beta|$ increases nonlinear effects. The nonlinearity is soft for $\beta<0$ and hard for $\beta>0$. 
The input BVP includes equations (1), (2), and (6). The proposed model of imperfect bonding is valid in the case of weakly-nonlinear properties at the interface. Equation (6) has an asymptotic nature and can be applied if, on its righthand side, the ratio of the second-to-first terms is of the order $10^{-1}$. The ratio $\sigma^{*} / \mu^{(1)}$ is equal to the elastic strain $\varepsilon$. In consequence, the following estimation for the admissible values of $\beta$ is obtained: $|\beta| \leq\left(10 \varepsilon^{2}\right)^{-1}$. In most solids, the elastic strains do not exceed $10^{-3}$, which implies $|\beta| \leq 10^{5}$.

It should be noted that BVP (1), (2), (6) allows different physical interpretations. Here we consider it in terms of the anti-plane shear waves, however, all the following mathematical results are also valid for electromagnetic waves in composites with dielectric inclusions.

3. Higher-order asymptotic homogenization. Let us introduce nondimensional variables $\bar{u}=u / U, \overline{\mathbf{n}}=\mathbf{n} / L, \bar{x}_{k}=x_{k} / L, k=1,2$, where $U$ is the displacement amplitude and $L$ is the wavelength. Taking into account that $\sigma^{*}=\mu^{(1)}\left(\partial u^{(1)} / \partial \mathbf{n}\right)$ at $\partial \Omega$, the input BVP (1), (2), (6) reads

$$
\begin{gathered}
\mu^{(n)} \nabla_{\bar{x}}^{2} \bar{u}^{(n)}=\rho^{(n)} L^{2} \frac{\partial^{2} \bar{u}^{(n)}}{\partial t^{2}}, \\
\mu^{(1)} \frac{\partial \bar{u}^{(1)}}{\partial \overline{\mathbf{n}}}=\mu^{(2)} \frac{\partial \bar{u}^{(2)}}{\partial \overline{\mathbf{n}}} \text { at } \partial \Omega, \\
\bar{u}^{(1)}-\bar{u}^{(2)}=\alpha \eta \frac{\partial \bar{u}^{(1)}}{\partial \overline{\mathbf{n}}}-\alpha \eta \delta\left(\frac{\partial \bar{u}^{(1)}}{\partial \overline{\mathbf{n}}}\right)^{3} \text { at } \partial \Omega,
\end{gathered}
$$

where $\eta=l / L, \delta=\beta(U / L)^{2}, \nabla_{\bar{x}}=L^{-1} \nabla_{x}$.

The ratio $U / L$ indicates the magnitude of the elastic strains. We suppose that the size $l$ of the unit cell is smaller than the wavelength $L$. Hence, the nondimensional variables $\eta$ and $\delta$ may be considered as natural small parameters characterising, accordingly, the rate of dispersion and the rate of nonlinearity. 
The obtained BVP (7)-(9) implies that both the matrix and the fibres exhibits linear elastic behaviour, while the nonlinearity is induced particularly by the imperfect bonding conditions at the interface. This model is valid, if the gradients of displacement are small enough (so that the geometrical and the physical nonlinearity of the material can be neglected). However, at the same time, the interface response is essentially nonlinear (thus, the parameter $\beta$ is sufficiently large). Such a statement of the problem give us a possibility to distinguish and to investigate, how the nonlinear bonding conditions between the components can affect the overall mechanical properties of the composite material. For the study of geometrical and physical nonlinearity we refer to Samsonov (2001), Porubov (2003), Andrianov et al. $(2013,2014)$.

In order to derive a macroscopic wave equation, the method of asymptotic homogenization is applied. Let us introduce so-called fast $y_{k}=\eta^{-1} \bar{x}_{k}$ and slow $\bar{x}_{k}=\bar{x}_{k}$ coordinate variables. The spatial derivatives are then given by

$$
\nabla_{\bar{x}}=\nabla_{\bar{x}}+\eta^{-1} \nabla_{y}
$$

where $\nabla_{y}=\left(\partial / \partial y_{1}\right) \mathbf{e}_{1}+\left(\partial / \partial y_{2}\right) \mathbf{e}_{2}$.

The solution is sought as the asymptotic expansion:

$$
\bar{u}^{(n)}=u_{0}\left(\bar{x}_{k}\right)+\eta u_{1}^{(n)}\left(\bar{x}_{k}, y_{k}\right)+\eta^{2} u_{2}^{(n)}\left(\bar{x}_{k}, y_{k}\right)+\ldots
$$

Here the first term $u_{0}$ represents the homogenized part of the displacement field; it varies "slowly" on the macrolevel and does not depend on the fast coordinates. The next terms $u_{i}^{(n)}, i=1,2,3, \ldots$, provide order $\eta^{i}$ corrections and describe local oscillations of the displacements within each unit cell. Since the composite structure is periodic, the functions $u_{i}^{(n)}$ satisfy the periodicity condition: 


$$
u_{i}^{(n)}\left(\bar{x}_{k}, y_{k}\right)=u_{i}^{(n)}\left(\bar{x}_{k}, y_{k} \pm 1\right), \quad i=1,2,3, \ldots
$$

Splitting the BVP (7)-(9) with respect to $\eta$, we obtain the recurrent sequence of local BVPs:

$$
\begin{gathered}
\mu^{(n)}\left(\nabla_{\bar{x}}^{2} u_{i-2}^{(n)}+2 \nabla_{\bar{x}} \cdot \nabla_{y} u_{i-1}^{(n)}+\nabla_{y}^{2} u_{i}^{(n)}\right)=\rho^{(n)} L^{2} \frac{\partial^{2} u_{i-2}^{(n)}}{\partial t^{2},} \\
\mu^{(1)}\left(\frac{\partial u_{i-1}^{(1)}}{\partial \overline{\mathbf{n}}}+\frac{\partial u_{i}^{(1)}}{\partial \mathbf{m}}\right)=\mu^{(2)}\left(\frac{\partial u_{i-1}^{(2)}}{\partial \overline{\mathbf{n}}}+\frac{\partial u_{i}^{(2)}}{\partial \mathbf{m}}\right) \text { at } \partial \Omega, \\
u_{i}^{(1)}-u_{i}^{(2)}=\alpha\left(\frac{\partial u_{i-1}^{(1)}}{\partial \overline{\mathbf{n}}}+\frac{\partial u_{i}^{(1)}}{\partial \mathbf{m}}\right)-\alpha \delta\left(\frac{\partial u_{i-1}^{(1)}}{\partial \overline{\mathbf{n}}}+\frac{\partial u_{i}^{(1)}}{\partial \mathbf{m}}\right)^{3} \text { at } \partial \Omega,
\end{gathered}
$$

where $i=1,2,3, \ldots ; u_{-1}^{(n)}=0 ; \partial / \partial \mathbf{m}$ is the normal derivative to $\partial \Omega$ written in terms of fast variables.

The periodicity condition (11) can be equivalently replaced by the condition of zero mean value over the unit cell domain (Bakhvalov and Panasenko, 1989):

$$
\iint_{\Omega_{0}^{(1)}} u_{i}^{(1)} d y_{1} d y_{2}+\iint_{\Omega_{0}^{(2)}} u_{i}^{(2)} d y_{1} d y_{2}=0
$$

The local problems (12) -(15) are considered within a specific unit cell of the composite structure (Fig. 2). In terms of the fast coordinates $y_{k}$, the size of the unit cell is unity with the radius of the inclusion $\sqrt{c^{(2)} / \pi}$, where $c^{(2)}$ is the volume fraction of the fibres and $0 \leq c^{(2)} \leq \pi / 4$. 


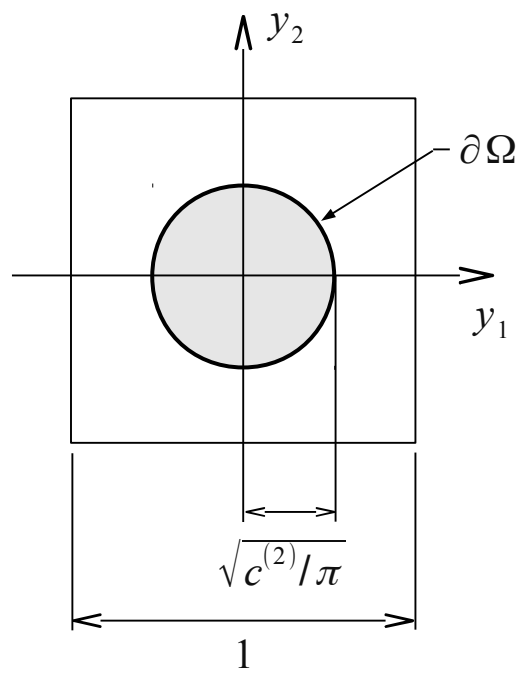

Fig.2. The unit cell.

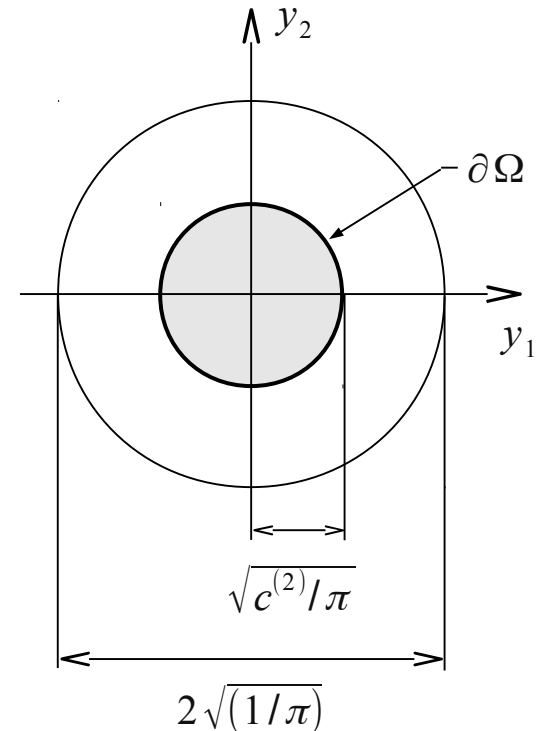

Fig. 3. Simplification of the geometrical shape of the unit cell.

Solution of the local problems may be obtained by different methods. Among them, we would like to point out the method of Rayleigh multipole-expansions, which was efficiently used for the determination of effective properties as well as for study of the wave propagation in periodic composite structures (Movchan et al., 2002; Parnell and Abrahams, 2006; William and Parnell, 2014). In this paper, we derive an approximate analytical solution of the local problem (12)-(15). Let us replace the outer square contour of the unit cell by a circle of the same area (Fig. 3). This simplification is well known in the theory of composites. In particular, it was used for the evaluation of effective properties by the method of composite cylinders assemblage and within generalised self consistent scheme (Christensen, 2005). The accuracy of such an approach is known to be good, when the volume fraction of the inclusions is relatively small. A numerical verification will be presented below.

Solutions of the nonlinear local problems (12)-(15) are sought through the following asymptotic expansion: 


$$
u_{i}^{(n)}=u_{i 0}^{(n)}+\delta u_{i 1}^{(n)}+O\left(\delta^{2}\right)
$$

The coefficients within (16) are determined explicitly using the computer algebra system "Maple". The term $u_{1}^{(n)}$ is evaluated with accuracy $O(\delta)$, the terms $u_{2}^{(n)}, u_{3}^{(n)}$ - with accuracy $O\left(\delta^{0}\right)$.

Next, we apply to equation (12) at $i=4$ the homogenizing operator $\iint(\cdot) d y_{1} d y_{2}$ over the unit cell domain. The term $u_{4}^{(n)}$ is eliminated by use of Green's theorem and taking into account conditions (11), (13) (Bakhvalov and Panasenko, 1989). As a result, the macroscopic nonlinear wave equation is obtained in the form:

$$
\mu_{1} \nabla_{\bar{x}}^{2} u_{0}+\frac{1}{3} \delta \mu_{2} \nabla_{\bar{x}} \cdot\left(\nabla_{\bar{x}} u_{0}\right)^{3}+\eta^{2} \mu_{3} \nabla_{\bar{x}}^{4} u_{0}+O\left(\delta^{2}+\eta^{4}\right)=\rho L^{2} \frac{\partial^{2} u_{0}}{\partial t^{2}}
$$

Reverting back to the dimension variables $u=u_{0} U, x_{k}=\bar{x}_{k} L$, equation (17) reads

$$
\mu_{1} \nabla_{x}^{2} u+\frac{1}{3} \beta \mu_{2} \nabla_{x} \cdot\left(\nabla_{x} u\right)^{3}+\eta^{2} L^{2} \mu_{3} \nabla_{x}^{4} u+O\left(\delta^{2}+\eta^{4}\right)=\rho \frac{\partial^{2} u}{\partial t^{2}}
$$

where $\mu_{1}, \mu_{2}, \mu_{3}$ are the effective coefficients. The parameter $\mu_{1}$ is the linear shear modulus; the parameters $\mu_{2}$ and $\mu_{3}$ account, respectively, for nonlinearity and for dispersive properties.

For the effective coefficients $\mu_{1}$ and $\mu_{2}$, explicit analytical formulas have been derived (see Appendix). The coefficient $\mu_{3}$ was evaluated by numerical integration over the unit cell. All calculations were performed using "Maple". It should be noted that $\mu_{1}, \mu_{2}, \mu_{3}$ are always positive.

The presence of the microstructure leads to the appearance in the macroscopic 
wave equation (18) of the higher-order gradient term $\eta^{2} L^{2} \mu_{3} \nabla_{x}^{4} u$. This term not only refines the low-frequency (acoustic) mode of interest but also generates a spurious high-frequency mode. The latter doesn't appear in the asymptotically identical equation

$$
\mu_{1} \nabla_{x}^{2} u+\frac{1}{3} \beta \mu_{2} \nabla_{x} \cdot\left(\nabla_{x} u\right)^{3}+\eta^{2} L^{2} \frac{\mu_{3}}{\mu_{1}} \rho \nabla_{x}^{2} \frac{\partial^{2} u}{\partial t^{2}}+O\left(\delta^{2}+\eta^{4}\right)=\rho \frac{\partial^{2} u}{\partial t^{2}}
$$

derived from (18) by the substitution $\nabla_{x}^{4} u=\left(\rho / \mu_{1}\right) \nabla_{x}^{2}\left(\partial^{2} u / \partial t^{2}\right)$.

The spurious high-frequency modes arising in refined long wave theories have been thoroughly investigated within the context of thin elastic structures, e.g. see Goldenveizer et al., 1993; Kaplunov et al., 1998, 2000; Pichugin et al., 2008 and references therein. The results of the aforementioned publications can be also extended to the long-wave models of periodic media (e.g., see Boutin and Auriault, 1993; Chen and Fish, 2001; Fish and Chen, 2004; Andrianov et al., 2008; Auriault and Boutin, 2012; Soubestre and Boutin, 2012) having a lot in common with those for thin structures, see also Craster et al., 2014.

The solution obtained in this paper is based on a geometrical simplification of the shape of the unit cell. In order to estimate the numerical accuracy of this approach, we consider the linear effective shear modulus $\mu_{1}$ in the case of perfect bonding $(\alpha=0)$. The expression obtained for $\mu_{1}$ reads

$$
\mu_{1}=\mu^{(1)} \frac{\left(1-c^{(2)}\right) \mu^{(1)}+\left(1+c^{(2)}\right) \mu^{(2)}}{\left(1+c^{(2)}\right) \mu^{(1)}+\left(1-c^{(2)}\right) \mu^{(2)}}
$$

Formula (19) may be obtained through a generalised self-consistent scheme (Christensen, 2005). It also coincides with the lower variational bound obtained by Hashin (1965). 
Table 1 . The effective shear modulus $\mu_{1} / \mu^{(1)}$ of a composite with perfectly bonded, perfectly rigid fibres $\left(\alpha=0, \mu^{(2)} / \mu^{(1)} \rightarrow \infty\right)$.

\begin{tabular}{|c|c|c|c|}
\hline$c^{(2)}$ & $\begin{array}{c}\text { Approximate } \\
\text { solution (19) }\end{array}$ & $\begin{array}{c}\text { Data by Perrins et } \\
\text { al. (1979) }\end{array}$ & $\begin{array}{c}\text { Approximation } \\
\text { error, \% }\end{array}$ \\
\hline 0.1 & 1.222 & 1.222 & 0.0 \\
0.2 & 1.500 & 1.500 & 0.0 \\
0.3 & 1.857 & 1.860 & 0.2 \\
0.4 & 2.333 & 2.351 & 0.8 \\
0.5 & 3.000 & 3.080 & 2.6 \\
0.6 & 4.000 & 4.342 & 7.9 \\
0.7 & 5.667 & 7.433 & 24.8 \\
0.74 & 6.692 & 11.01 & 39.2 \\
0.76 & 7.333 & 15.44 & 52.5 \\
0.77 & - & 20.43 & - \\
0.78 & - & 35.93 & - \\
\hline
\end{tabular}

In Table 1, the analytical solution (19) is compared with theoretical results obtained by Perrins et al. (1979) for a composite material with perfectly rigid fibres $\left(\mu^{(2)} / \mu^{(1)} \rightarrow \infty\right)$. These results are known to converge. This example exhibits the highest concentrations of local stress, the error of formula (19) then reaching a maximum. We remark that a decrease in the rigidity of fibres will improve the accuracy of the approximation.

It can be seen that expression (19) provides a good accuracy for $c^{(2)}<0.5 \ldots 0.6$. However, the error grows rapidly at $c^{(2)}>0.7$, when the gaps between neighbouring fibres narrow and high gradients of the local fields are induced. In the latter case, the shape of the unit cell can no longer be neglected and the spacial arrangement of the fibres must be taken into account. However, it should be noted that for most 
real composite materials the volume fraction of the fibres does not exceed 0.4...0.5. The approximate solutions obtained in the present paper can therefore be successfully and confidently applied.

The macroscopic wave equation (18) is valid for long waves, i.e. when $\eta=l / L<1$. Below, a dispersion relation evaluated from equation (18) is compared with the exact solution obtained by the Floquet-Bloch theory.

Let us consider the linear case $\left(\mu_{2}=0\right)$ and the harmonic wave

$$
u=U \exp (i \mathbf{k} \cdot \mathbf{x}) \exp (-i \omega t)
$$

where $U$ is the amplitude; $\omega$ is the frequency; $\mathbf{k}$ is the wave vector, $\mathbf{k}=k_{1} \mathbf{e}_{1}+k_{2} \mathbf{e}_{2}$; $\mathbf{x}=x_{1} \mathbf{e}_{1}+x_{2} \mathbf{e}_{2} ; k_{1}, k_{2}$ projections of the wave vector $\mathbf{k}$ onto the coordinate axes, $k_{1}=k \cos \varphi, \quad k_{2}=k \sin \varphi$, where $k=|\mathbf{k}|=\sqrt{\left(k_{1}^{2}+k_{2}^{2}\right)}=2 \pi / L ;$ with $\varphi$ the angel between the axis $x_{1}$ and the wave vector $\mathbf{k}$.

Substituting expression (20) into equation (18), we obtain the dispersion relation:

$$
\omega^{2}=\omega_{0}^{2}\left[1-4 \pi^{2} \frac{\mu_{3}}{\mu_{1}} \eta^{2}+O\left(\eta^{4}\right)\right]
$$

where $\omega_{0}=v_{0} k ; v_{0}$ is the effective phase velocity in the linear long-wave limit, $v_{0}=\sqrt{\mu_{1} / \rho}$. The parameter $\eta$ is related to the modulus $k$ of the wave vector through $\eta=l / L=k l /(2 \pi)$.

For the composite structure under consideration (Fig. 1), the anti-plane shear problem is transversely isotropic. However, this is true only for the static deformation. In the dynamic case, the isotropic behaviour occurs only in the longwave limit, when the wavelength is essentially larger than the size of the microstructure, $l / L \rightarrow 0$ and $\omega \rightarrow 0$. If the wavelength decreases (i.e., the frequency 
increases), the composite material exhibits anisotropic properties. The parameters of high-frequency elastic waves depend on the direction of the wave vector, which is governed by the angel $\varphi$. Generally, this effect is well known and can be observed from the dispersion diagrams for anti-plane shear waves derived by the Floquet-Bloch method (Andrianov et al., 2008; Kushwaha et al., 1994). In this paper, the simplification of the geometrical shape of the unit cell presented in Fig. 3 implies the axial symmetry of the local problems. Therefore, the derived approximate solution is transversely isotropic and the macroscopic wave equation (18) is invariant to the direction of the wave propagation.

In Fig. 4, the asymptotic solution (21) (dashed curves) is compared with the exact dispersion relation (solid curves) evaluated by the Floquet-Bloch method (Andrianov et al., 2008) for different directions of the wave vector: orthogonal ( $\varphi=0)$ and diagonal $(\varphi=\pi / 4)$ with respect to the unit cell. Numerical results are presented for a composite material consisting of an aluminium matrix ( $\left.\mu^{(1)}=27.9 \mathrm{GPa}, \quad \rho^{(1)}=2700 \mathrm{~kg} / \mathrm{m}^{3}\right)$ and nickel fibres $\left(\mu^{(2)}=75.4 \mathrm{GPa}, \rho^{(2)}=8940\right.$ $\left.\mathrm{kg} / \mathrm{m}^{3}\right)$, with fibre volume fraction $c^{(2)}=0.4$. It can be observed that the homogenized wave equation (18) provides good accuracy at $\eta<0.4$.

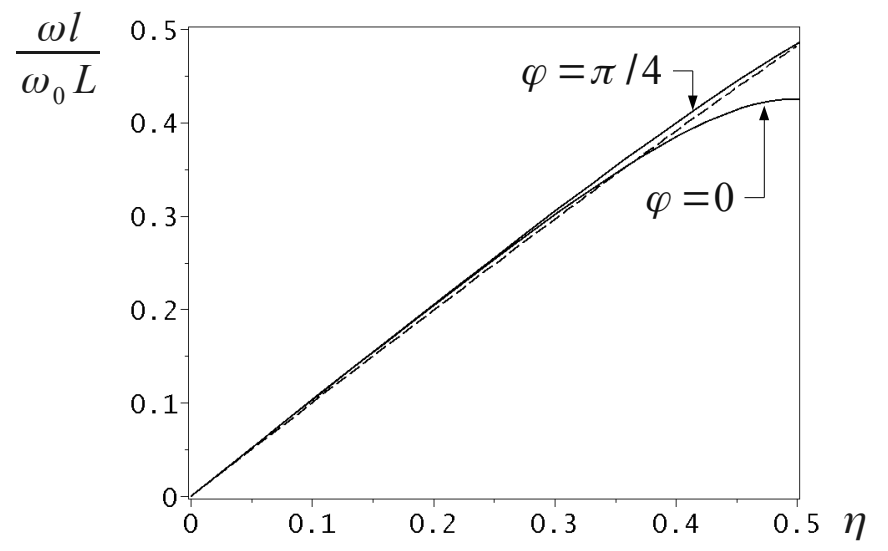

Fig. 4. Acoustic branches of the dispersion curves in the linear case. 
4. Analytical solution for stationary waves. We consider a stationary plane wave propagating with permanent shape and velocity in the direction of the wave vector $\mathbf{k}$. In such a case the solution meets the following condition:

$$
u(\mathbf{x}, t)=u(\xi),
$$

where $\xi$ is the propagation coordinate, $\xi=\mathbf{e}_{k} \cdot \mathbf{x}-v t ; v$ is the phase velocity and $\mathbf{e}_{k}$ is the unit wave vector, given by $\mathbf{e}_{k}=\mathbf{e}_{1} \cos \varphi+\mathbf{e}_{2} \sin \varphi$.

Let us define the strain of the wave profile as follows

$$
f=\frac{d u}{d \xi}
$$

and introduce non-dimensional variables $\bar{f}=f / F, \quad \xi=\xi / L$. Here $F$ is the amplitude of the strain wave and $L$ is the wavelength. Substituting expressions (22), (23) into equation (18) and integrating with respect to $\xi$, one obtains the nondimensional equation of a cubically nonlinear anharmonic oscillator:

$$
\frac{d^{2} \bar{f}}{d \zeta^{2}}+a \bar{f}+b \bar{f}^{3}+c=0
$$

where $a=\mu_{1}\left(1-v^{2} / v_{0}^{2}\right) /\left(\mu_{3} \eta^{2}\right), \quad b=\beta F^{2} \mu_{2} /\left(3 \mu_{3} \eta^{2}\right), \quad c \quad$ is the constant of integration.

The natural assumption that the displacement $u$ remains bounded imposes the condition of a zero mean strain over the wave period, thus

$$
\int_{0}^{1} \bar{f}(\xi) d \zeta=0
$$


Condition (25) allows evaluation of the constant $c$. In the problem under consideration we obtain $c=0$.

Equation (24) represents a well-known reduction of the famous modified Korteweg-de Vries equation (e.g., see Drazin and Jonson, 1989; Christov et al., 1996; Maugin, 1999; Porubov, 2003). In this case propagation of stationary nonlinear waves is possible in the case of a balance state between the effects of nonlinearity and dispersion. This means that all the terms of equation (24) have to be of the same asymptotic order. Let us establish the underlying scaling.

The first term in equation (24) is of the order $O(1)$, whereas the asymptotic order of the second term is given by $\left(1-v^{2} / v_{0}^{2}\right) \eta^{-2}$. Then

$$
\left|1-v^{2} / v_{0}^{2}\right| \sim \eta^{2}
$$

implying that the studied wave speed $v$ has to be close to the shear speed $v_{0}$ predicted by the associated linear theory. Therefore we get for a typical wavelength

$$
L \sim \frac{l}{\sqrt{\left|1-v^{2} / v_{0}^{2}\right|}} \gg l
$$

Formula (26) means that we deal with long wave phenomena in line with the classical $\mathrm{KdV}$ setup originating from nonlinear analysis of shallow water waves.

The asymptotic order of the third term in (24) depends on $\beta F^{2} / \eta^{2}$. Taking into account $F \sim U / L$, and $\delta=\beta(U / L)^{2}$ (see Section 2), we obtain that the third term of equation (24) is also $O(1)$. The typical magnitudes of the parameters are as follows: $\eta^{2} \sim 10^{-1}, \delta \sim 10^{-1},|\beta| \sim 10^{5}, F \sim 10^{-3}$.

The exact analytical solutions of equation (24) can be derived in terms of elliptic functions (see, for example, Erofeev (2003)).The type of solution depends upon the signs of the coefficients $a$ and $b$, so we obtain different results for soft 
and for hard nonlinearity.

In the case of soft nonlinearity $(\beta<0, b<0)$, the exact periodical solution of Eq. (24) is:

$$
\bar{f}=\frac{1}{2} \operatorname{sn}(\kappa \zeta, s)
$$

where $\kappa$ is the propagation variable, $\kappa=4 K(s), \operatorname{sn}(\cdot)$ is the elliptic sine, $K(s)$ is the complete elliptic integral of the first kind (Abramowitz and Stegun, 1965), $s$ is the modulus of the elliptic functions that is determined form the transcendental equation

$$
s^{2} K(s)^{2}=-\frac{\beta F^{2} \mu_{2}}{384 \eta^{2} \mu_{3}} .
$$

For the phase velocity $v$ we obtain:

$$
\frac{v^{2}}{v_{0}^{2}}=1-16\left(1+s^{2}\right) K(s)^{2} \frac{\mu_{3}}{\mu_{1}} \eta^{2}
$$

In the case of hard nonlinearity $(\beta>0, b>0)$, the solution takes the following form:

$$
\begin{gathered}
\bar{f}=\frac{1}{2} \operatorname{cn}(\kappa \zeta, s) . \\
s^{2} K(s)^{2}=\frac{\beta F^{2} \mu_{2}}{384 \eta^{2} \mu_{3}}, \\
\frac{v^{2}}{v_{0}^{2}}=1-16\left(1-2 s^{2}\right) K(s)^{2} \frac{\mu_{3}}{\mu_{1}} \eta^{2} .
\end{gathered}
$$


We remark that equations (28) and (31) transform into each other, if the sign of the parameter $\beta$ changes.

\section{Analysis of the solution and numerical results}

In the problem under consideration, the type of nonlinearity (soft at $\beta<0$ or hard at $\beta>0$ ) has a crucial influence upon the propagation mode of elastic strain waves. The shapes of the wave profile are displayed in Fig. 5 employing expressions (27) and (30). The magnitude of the modulus $s$ determines the intensity of nonlinear effects.

The limit $s=0$ corresponds to the purely linear case:

$$
\bar{f}=\frac{1}{2} \sin (2 \pi \zeta) \text { at } \beta<0 ; \quad \bar{f}=\frac{1}{2} \cos (2 \pi \zeta) \text { at } \beta>0 ; \quad \frac{v^{2}}{v_{0}^{2}}=1-4 \pi^{2} \frac{\mu_{3}}{\mu_{1}} \eta^{2} \text {. }
$$

At the opposite limit, $s=1$, solutions (27)-(29) and (30)-(32) describe localised solitary waves. In the case of soft nonlinearity, a shock (so-called kink) strain wave appears (Fig. 6, $a$ ):

$$
\bar{f}=\frac{1}{2} \operatorname{th}\left(\frac{\zeta}{\Delta}\right), \quad \Delta^{2}=-\frac{24 \mu_{3} \eta^{2}}{F^{2} \beta \mu_{2}}, \frac{v^{2}}{v_{0}^{2}}=1-\frac{2 \mu_{3} \eta^{2}}{\mu_{1} \Delta^{2}}, \quad \beta<0 .
$$

Here the parameter $\Delta$ can be treated as the width of the localised wave.

The kink wave propagates with a velocity lower than $v_{0}$ associated with the linear long-wave limit: $v<v_{0}$. This is the so-called subsonic mode. The increase in the amplitude $F$ leads to a decrease in the width $\Delta$ and the velocity $v$ of the wave. 


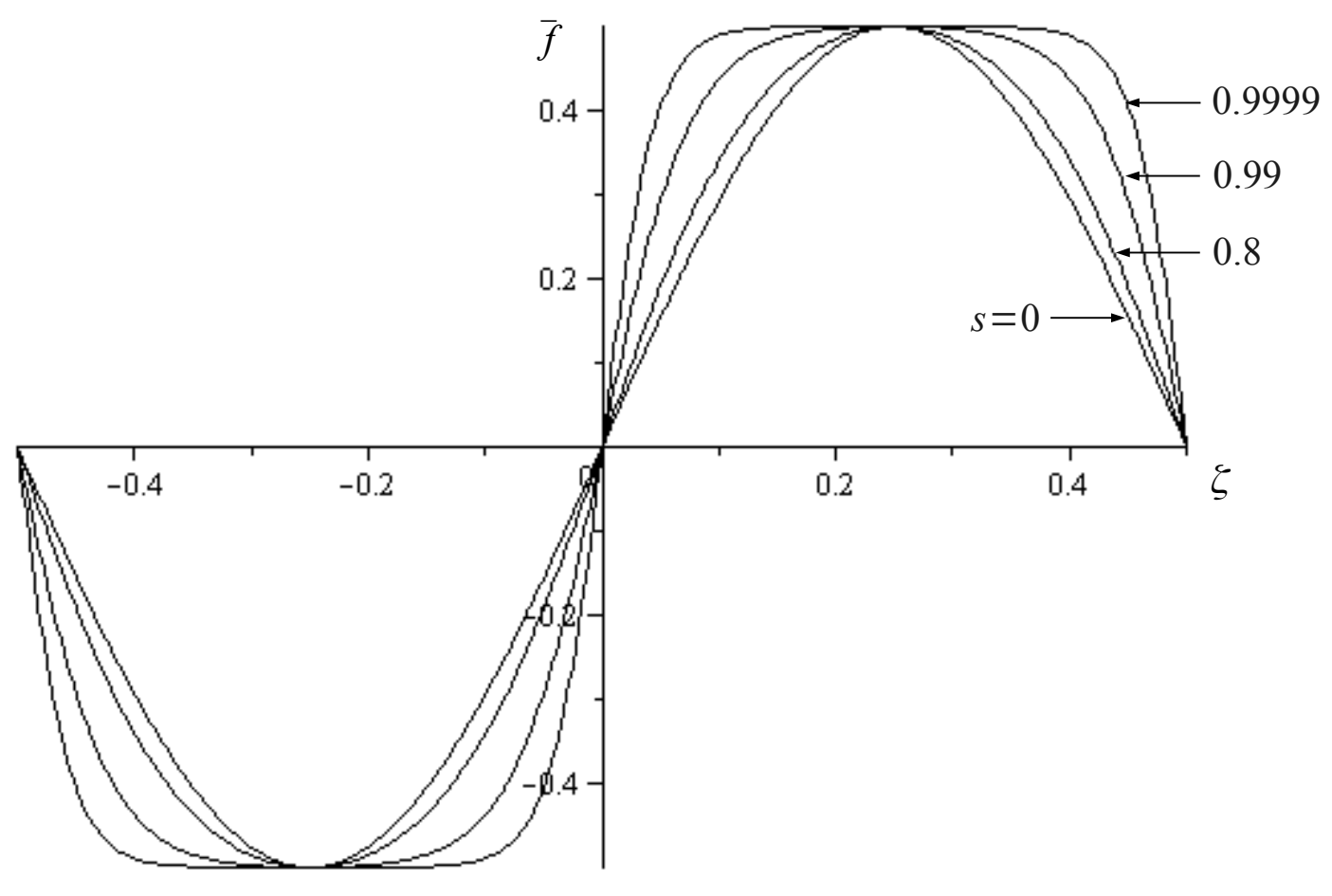

$a$

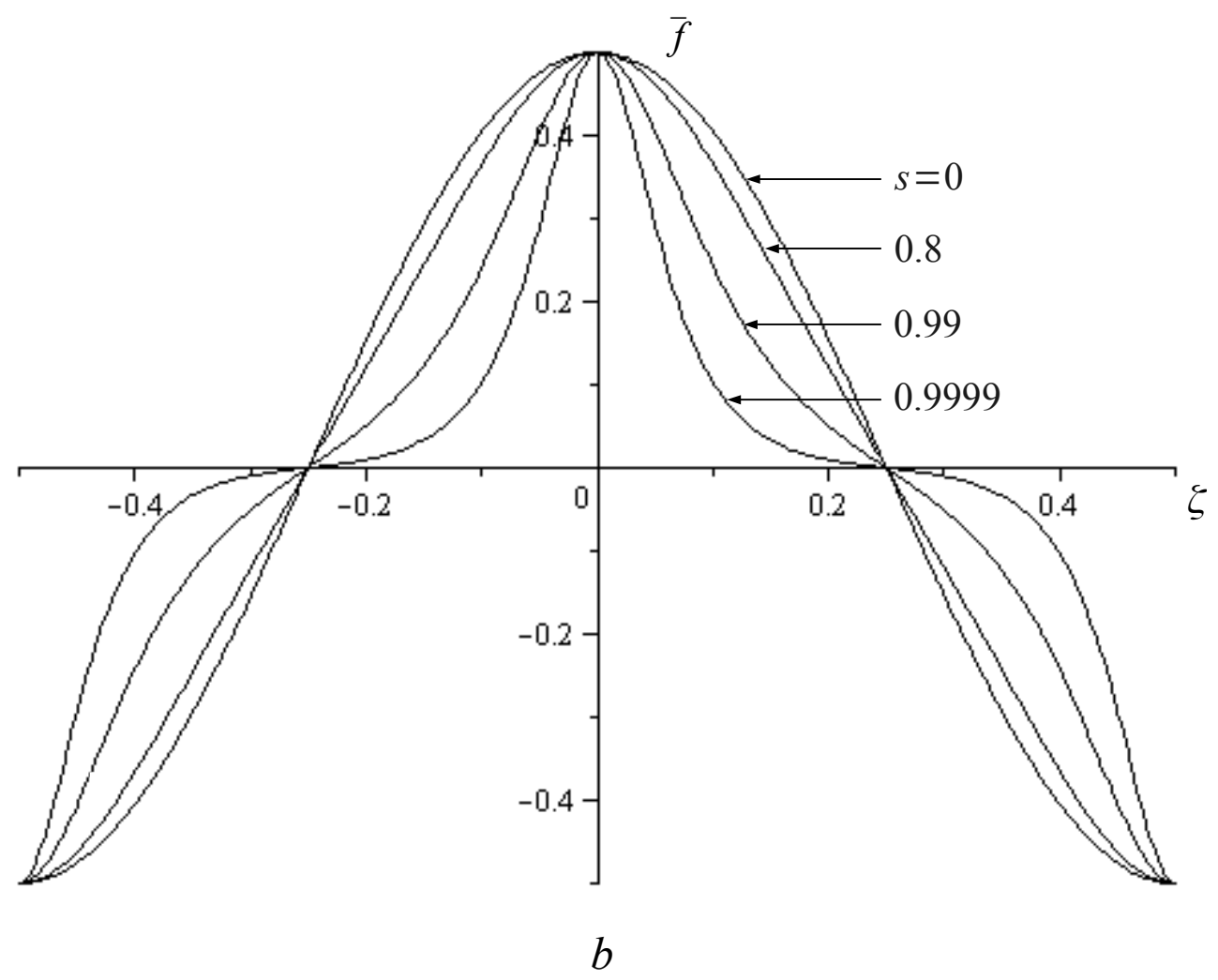

Fig. 5. Periodic nonlinear elastic strain waves;

$a$ - soft nonlinearity $(\beta<0), b-$ hard nonlinearity $(\beta>0)$. 


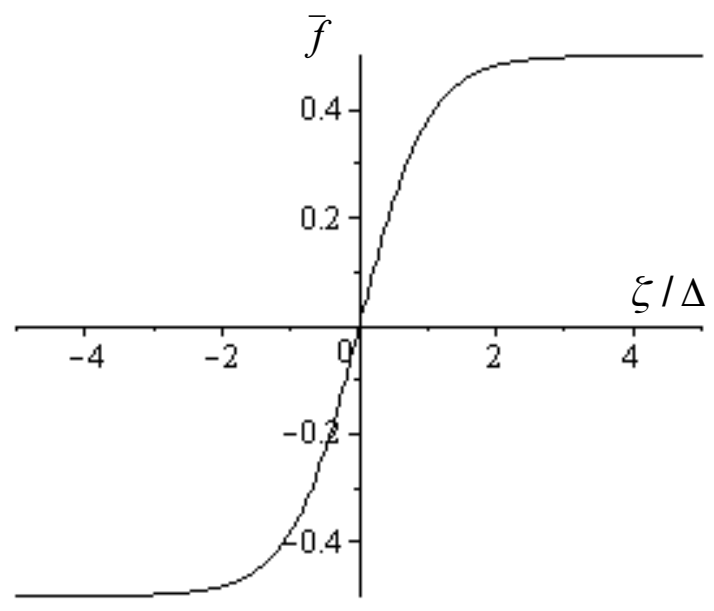

$a$

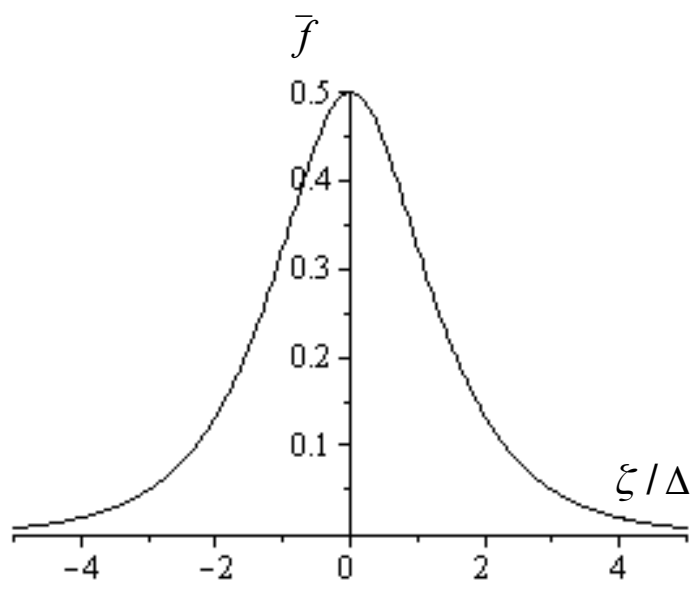

$b$

Fig. 6. Localised nonlinear elastic strain waves;

$a$ - soft nonlinearity $(\beta<0), b-$ hard nonlinearity $(\beta>0)$.

If nonlinearity is hard, the localised solution takes the form of a bell-shaped wave (Fig. 6, $b$ ):

$$
\bar{f}=\frac{1}{2 \operatorname{ch}(\zeta / \Delta)}, \quad \Delta^{2}=\frac{24 \mu_{3} \eta^{2}}{F^{2} \beta \mu_{2}}, \quad \frac{v^{2}}{v_{0}^{2}}=1+\frac{\mu_{3} \eta^{2}}{\mu_{1} \Delta^{2}}, \quad \beta>0 .
$$

In this case a supersonic propagation mode is realised, i.e. $v>v_{0}$. When the amplitude $F$ grows, the width $\Delta$ decreases and the velocity $v$ increases.

As an illustrative example, let us consider numerical results for a composite material consisting of the aluminium matrix $\left(\mu^{(1)}=27.9 \mathrm{GPa}, \rho^{(1)}=2700 \mathrm{~kg} / \mathrm{m}^{3}\right)$ and nickel fibres $\left(\mu^{(2)}=75.4 \mathrm{GPa}, \rho^{(2)}=8940 \mathrm{~kg} / \mathrm{m}^{3}\right)$. The volume fraction of the fibres is $c^{(2)}=0.4$. The following magnitudes of the bonding parameters are assumed: $\alpha=0.1,|\beta|=10^{5}$. Basing on the solutions presented in Section 3, the effective elastic coefficients are evaluated: $\mu_{1}=33.1 \mathrm{GPa}, \mu_{2}=21.6 \mathrm{GPa}, \mu_{3}=0.119 \mathrm{GPa}$.

The dependence of the phase velocity upon the modulus $s$ is presented in Fig. 7. In the case of soft nonlinearity $(\beta<0)$, a subsonic propagating mode is 
realised: $v<v_{0}$. For hard nonlinearity $(\beta>0)$, the subsonic mode $\left(v<v_{0}\right)$ occurs at $s^{2}<1 / 2$, while the supersonic mode $\left(v>v_{0}\right)$ is observed at $s^{2}>1 / 2$. Here the threshold value $s_{0}^{2}=1 / 2$ is determined by considering qualitative properties of the analytical solution (31) and is independent from physical characteristics of the material and direction of propagation.

Fig. 8 displays the parametric dependence of the modulus $s$ on the amplitude $F$ and the dispersion parameter $\eta$. These results have been obtained through numerical solution of equations (28), (31) using "Maple". The domain of elastic strains is restricted by $F \leq 10^{-3}$, a regime typical for most engineering materials.

The presented results show how the phenomena of nonlinearity and dispersion compensate each other. The increase of the amplitude $F$ (at a fixed value of $\eta$ ) leads to the growing of the modulus $s$ and, therefore, the intensity of nonlinear effects increases. In contrary, the decrease of the wavelength and the increase in $\eta$ (at a fixed $F$ ) is followed by the decrease of the modulus $s$, so the influence of nonlinearity is reduced.

As may be observed from Figs. 5, 7, if $s>0.6$ nonlinearity has a critical and fundamental influence on both the wave shape and velocity. In this case, $\eta<0.2$ and, consequently, the solution can be evaluated utilising long-wave approach. On the other hand, the homogenized equation (18) lacks accuracy for $\eta>0.4$ (see Fig. 4). However, in this case $s<0.34$, which means that the wave shape and velocity are very close to the linear case and, consequently, an approximate solution may be found utilising the linear theory. This analysis is particularly important, helping to estimate the domain of practical applicability of linear and nonlinear approaches. 


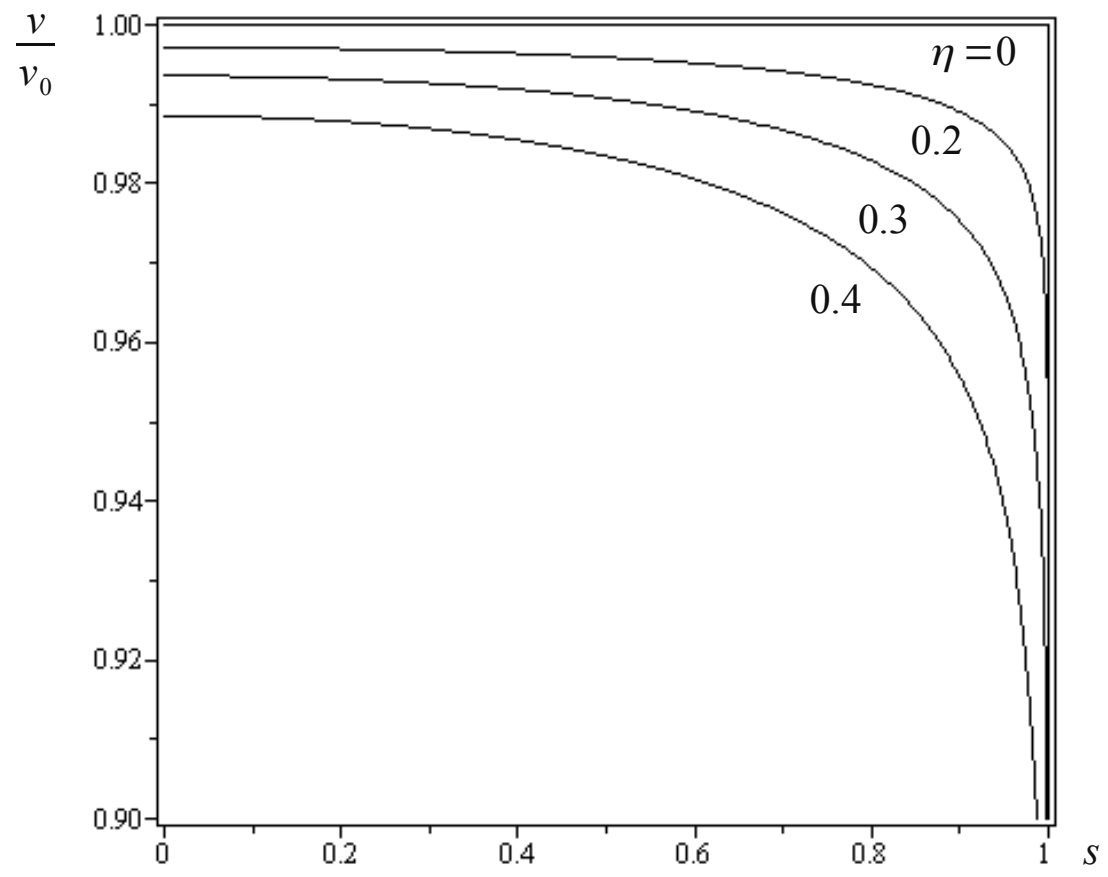

$a$

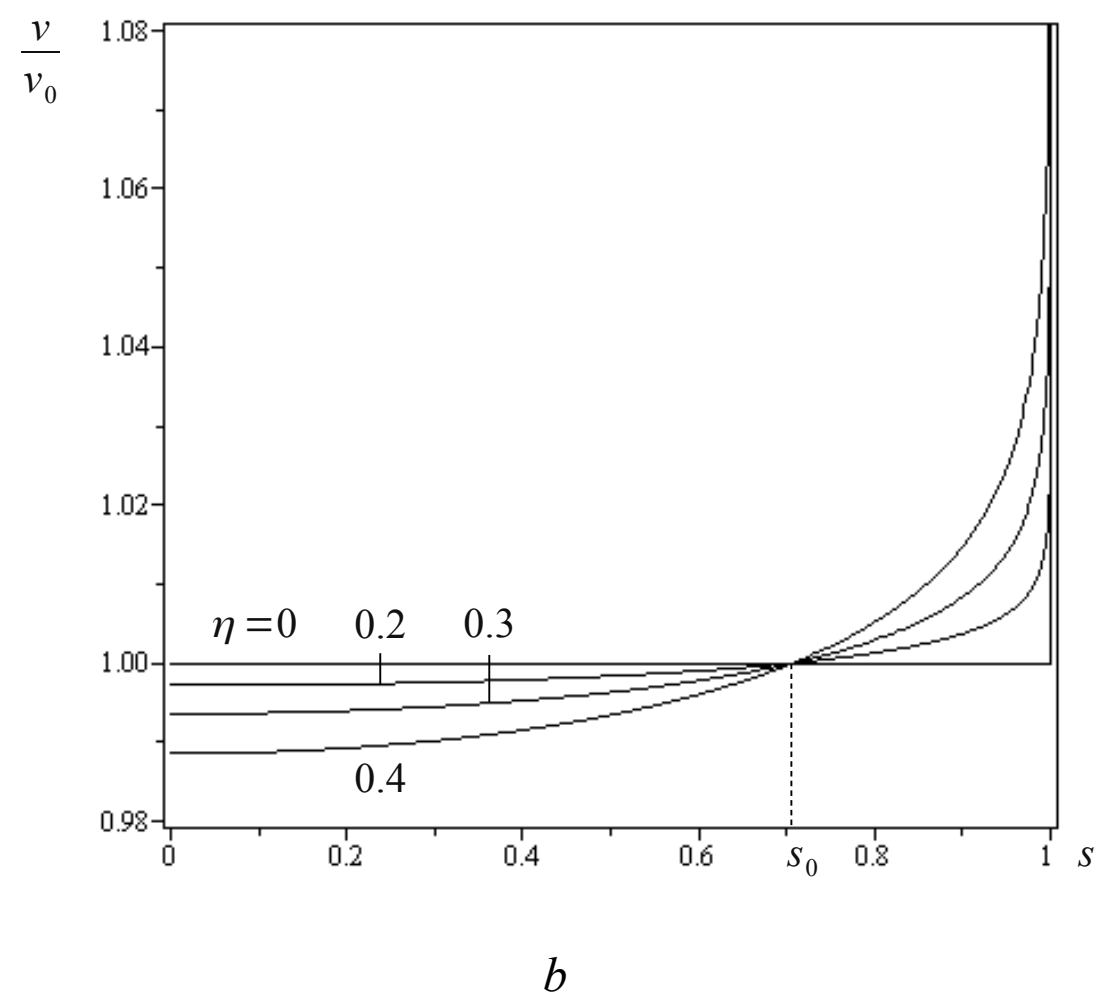

Fig. 7. Phase velocity of nonlinear elastic strain waves; $a$ - soft nonlinearity $(\beta<0), b-$ hard nonlinearity $(\beta>0)$. 


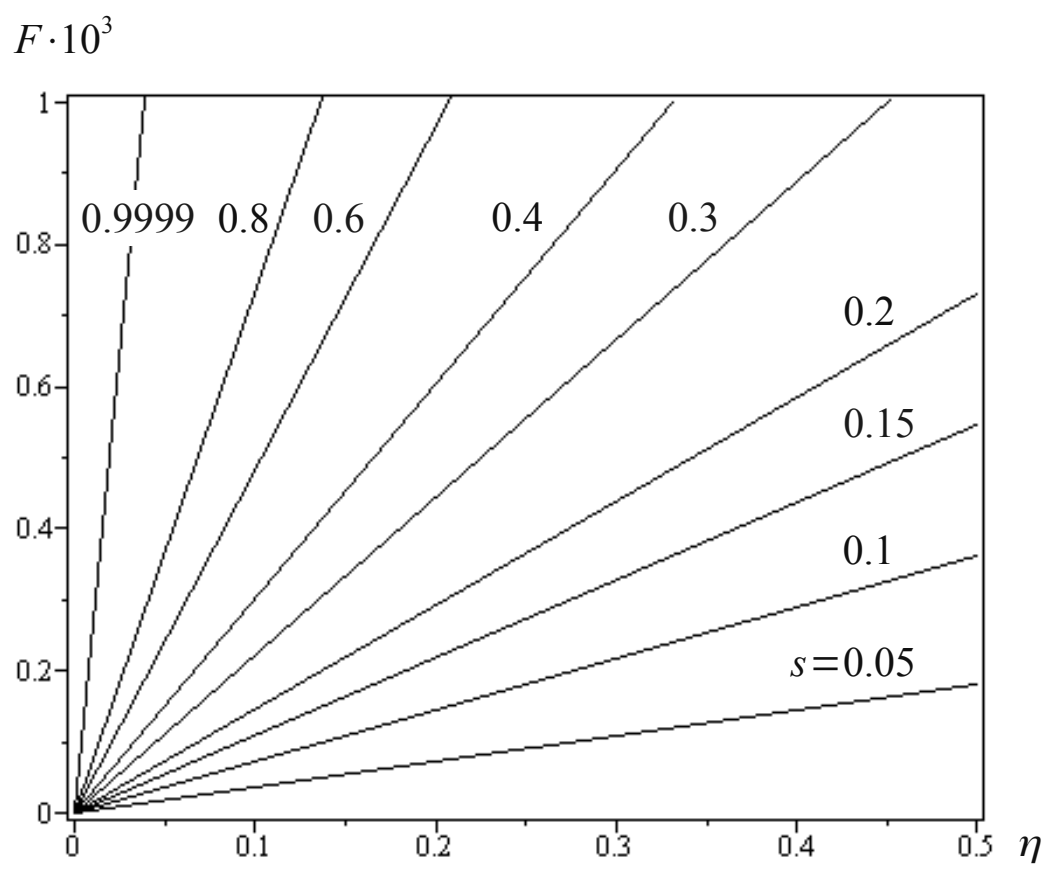

Fig. 8. The modulus $s$ characterising the intensity of nonlinear effects.

6. Conclusions. The paper presents analytical solutions that describe the propagation of nonlinear elastic anti-plane shear waves in a unidirectional fibrereinforced composite material with imperfect bonding between constitutive components. It should be emphasised that this type of nonlinearity has a particularly strong influence upon the propagation mode and the shape of the strain waves. In the case of soft nonlinearity, localised shock (kink) waves appear, while in respect of materials with hard nonlinearity the localised solution takes the form of bell-shaped waves.

The analysis allowed us to estimate the domain of applicability of the different approximate theories used for the modelling of elastic waves in heterogeneous solids. It is shown that nonlinear waves can be adequately described within the long-wave framework (such as the asymptotic homogenization method or Cosserat-type higher-order continuum models). When dealing with the propagation of short waves, with wave length commensurable with the scale of the microstructure, nonlinear effects become very small. In such a case, an approximate solution may be obtained using the linear Floquet-Bloch theory. 
The conclusion presented above is true, if the strain amplitude $F$ does not exceed $10^{-3}$, which is typical for solids. However, some materials and structures (e.g., elastometers, DNA molecules, nanotubes, atomic chains) can allow enormously high elastic strains ( $F=10^{-2} \ldots 10^{-1}$ or even higher). In such a case, short waves can not be considered in the framework of the linear elasticity, so both the effects of nonlinearity and high-dispersion (including phononic band gaps) must be taken into account simultaneously.

The results presented in the paper can be applied to facilitate the development of new efficient methods of acoustic diagnostic and non-destructive testing in various branches of engineering. Measuring the characteristics of nonlinear waves allows us to receive much more precise information about the internal structure and defects of solids. This is sometimes that may be not possible within a linear framework.

Finally, we remark that the propagation of localised nonlinear waves is accompanied by an essential concentration of mechanical energy. The obtained solutions for solitary strain waves can help in the development of new criteria for the dynamic failure of heterogeneous materials and structures.

Acknowledgement. The authors are grateful to anonymous referees, whose valuable comments helped to improve the paper. 


$$
\begin{gathered}
\mu_{1}=\left[\mu^{(1)}\left(c^{(2)}\right)^{2} \pi^{3 / 2}\left(\mu^{(2)}\right)^{2}+2\left(c^{(2)}\right)^{2} \pi^{3 / 2} \mu^{(2)}\left(\mu^{(1)}\right)^{2}-\mu^{(1)}\left(c^{(2)}\right)^{4} \pi^{3 / 2}\left(\mu^{(2)}\right)^{2}+\right. \\
2\left(c^{(2)}\right)^{4} \pi^{(3 / 2)} \mu^{(2)}\left(\mu^{(1)}\right)^{2}-\left(c^{(2)}\right)^{4} \pi^{3 / 2}\left(\mu^{(1)}\right)^{3}+\left(c^{(2)}\right)^{2} \pi^{3 / 2}\left(\mu^{(1)}\right)^{3}+ \\
2 \mu^{(1)} \alpha\left(\mu^{(2)}\right)^{2} \pi^{2}\left(c^{(2)}\right)^{3 / 2}+2 \alpha \mu^{(2)} \pi^{2}\left(c^{(2)}\right)^{3 / 2}\left(\mu^{(1)}\right)^{2}-\mu^{(1)} \alpha^{2}\left(\mu^{(2)}\right)^{2} \pi^{5 / 2}\left(c^{(2)}\right)^{3}+ \\
\left.\mu^{(1)} \alpha^{2}\left(\mu^{(2)}\right)^{2} c^{(2)} \pi^{5 / 2}+2 \mu^{(1)} \alpha\left(\mu^{(2)}\right)^{2} \pi^{2}\left(c^{(2)}\right)^{7 / 2}-2 \alpha \mu^{(2)}\left(c^{(2)}\right)^{7 / 2} \pi^{2}\left(\mu^{(1)}\right)^{2}\right] / \\
{\left[2 \alpha \mu^{(2)} \pi^{2}\left(c^{(2)}\right)^{3 / 2} \mu^{(1)}+2 \alpha \mu^{(2)}\left(c^{(2)}\right)^{7 / 2} \pi^{2} \mu^{(1)}-2\left(\mu^{(2)}\right)^{2}\left(c^{(2)}\right)^{3} \pi^{3 / 2}+\right.} \\
2\left(c^{(2)}\right)^{2} \pi^{3 / 2} \mu^{(2)} \mu^{(1)}-2\left(c^{(2)}\right)^{4} \pi^{3 / 2} \mu^{(2)} \mu^{(1)}+2 \alpha\left(\mu^{(2)}\right)^{2} \pi^{2}\left(c^{(2)}\right)^{3 / 2}+ \\
\alpha^{2}\left(\mu^{(2)}\right)^{2} \pi^{5 / 2}\left(c^{(2)}\right)^{3}+\alpha^{2}\left(\mu^{(2)}\right)^{2} c^{(2)} \pi^{5 / 2}-2 \alpha\left(\mu^{(2)}\right)^{2} \pi^{2}\left(c^{(2)}\right)^{7 / 2}+2\left(\mu^{(1)}\right)^{2}\left(c^{(2)}\right)^{3} \pi^{3 / 2}+ \\
\left(c^{(2)}\right)^{2} \pi^{3 / 2}\left(\mu^{(2)}\right)^{2}+\left(c^{(2)}\right)^{4} \pi^{3 / 2}\left(\mu^{(2)}\right)^{2}+\left(c^{(2)}\right)^{4} \pi^{3 / 2}\left(\mu^{(1)}\right)^{2}+\left(c^{(2)}\right)^{2} \pi^{3 / 2}\left(\mu^{(1)}\right)^{2}+ \\
\left.4 \alpha \mu^{(2)} \pi^{2}\left(c^{(2)}\right)^{5 / 2} \mu^{(1)}+2 \alpha^{2}\left(\mu^{(2)}\right)^{2} \pi^{5 / 2}\left(c^{(2)}\right)^{2}\right] \\
\mu_{2}=36 \alpha \mu^{(1)} \pi\left(c^{(2)}\right)^{5 / 2}\left(\mu^{(2)}\right)^{4} / \\
{\left[-4\left(c^{(2)}\right)^{5} \pi^{1 / 2}\left(\mu^{(2)}\right)^{4}+\left(c^{(2)}\right)^{6} \pi^{1 / 2}\left(\mu^{(1)}\right)^{4}+\right.} \\
\left(c^{(2)}\right)^{6} \pi^{1 / 2}\left(\mu^{(2)}\right)^{4}+12 c^{(2)}\left(\mu^{(2)}\right)^{3} \alpha^{2} \pi^{3 / 2} \mu^{(1)}+6 c^{(2)}\left(\mu^{(2)}\right)^{2} \alpha^{2} \pi^{3 / 2}\left(\mu^{(1)}\right)^{2}+
\end{gathered}
$$

$24\left(c^{(2)}\right)^{2}\left(\mu^{(2)}\right)^{2} \alpha^{2} \pi^{3 / 2}\left(\mu^{(1)}\right)^{2}+36\left(c^{(2)}\right)^{3}\left(\mu^{(2)}\right)^{2} \alpha^{2} \pi^{3 / 2}\left(\mu^{(1)}\right)^{2}+24\left(c^{(2)}\right)^{2}\left(\mu^{(2)}\right)^{3} \alpha^{2} \pi^{3 / 2} \mu^{(1)}-$ $24\left(c^{(2)}\right)^{4}\left(\mu^{(2)}\right)^{3} \alpha^{2} \pi^{3 / 2} \mu^{(1)}+4\left(c^{(2)}\right)^{5} \pi^{1 / 2}\left(\mu^{(1)}\right)^{4}+6\left(c^{(2)}\right)^{4} \pi^{1 / 2}\left(\mu^{(2)}\right)^{4}-$$$
12\left(c^{(2)}\right)^{4} \pi^{1 / 2}\left(\mu^{(1)} \mu^{(2)}\right)^{2}-8\left(c^{(2)}\right)^{5} \pi^{1 / 2}\left(\mu^{(1)}\right)^{3} \mu^{(2)}+8\left(c^{(2)}\right)^{3} \pi^{1 / 2} \mu^{(2)}\left(\mu^{(1)}\right)^{3}-
$$$$
4\left(c^{(2)}\right)^{6} \pi^{1 / 2}\left(\mu^{(2)}\right)^{3} \mu^{(1)}-4\left(c^{(2)}\right)^{6} \pi^{1 / 2} \mu^{(2)}\left(\mu^{(1)}\right)^{3}+\left(c^{(2)}\right)^{2} \pi^{1 / 2}\left(\mu^{(2)}\right)^{4}+
$$$$
\alpha^{4}\left(\mu^{(2)}\right)^{4} \pi^{5 / 2}+6\left(c^{(2)}\right)^{2} \pi^{1 / 2}\left(\mu^{(1)} \mu^{(2)}\right)^{2}+24\left(c^{(2)}\right)^{4}\left(\mu^{(2)}\right)^{2} \alpha^{2} \pi^{3 / 2}\left(\mu^{(1)}\right)^{2}-
$$$$
12\left(c^{(2)}\right)^{5}\left(\mu^{(2)}\right)^{3} \alpha^{2} \pi^{3 / 2} \mu^{(1)}+6\left(c^{(2)}\right)^{5}\left(\mu^{(2)}\right)^{2} \alpha^{2} \pi^{3 / 2}\left(\mu^{(1)}\right)^{2}+12\left(\mu^{(2)}\right)^{3} \pi \alpha\left(c^{(2)}\right)^{3 / 2} \mu^{(1)}+
$$$$
16\left(\mu^{(2)}\right)^{3} \pi^{2} \alpha^{3}\left(c^{(2)}\right)^{3 / 2} \mu^{(1)}+12\left(\mu^{(2)}\right)^{2} \pi \alpha\left(c^{(2)}\right)^{3 / 2}\left(\mu^{(1)}\right)^{2}+4 \mu^{(2)} \pi \alpha\left(c^{(2)}\right)^{3 / 2}\left(\mu^{(1)}\right)^{3}+
$$$$
4\left(\mu^{(2)}\right)^{3} \pi^{2} \alpha^{3}\left(c^{(2)}\right)^{9 / 2} \mu^{(1)}-24\left(\mu^{(2)}\right)^{2} \pi \alpha\left(c^{(2)}\right)^{9 / 2}\left(\mu^{(1)}\right)^{2}+16 \mu^{(2)} \pi \alpha\left(c^{(2)}\right)^{9 / 2}\left(\mu^{(1)}\right)^{3}+
$$$$
24\left(\mu^{(2)}\right)^{3} \pi^{2} \alpha^{3}\left(c^{(2)}\right)^{5 / 2} \mu^{(1)}+16 \mu^{(2)} \pi \alpha\left(c^{(2)}\right)^{5 / 2}\left(\mu^{(1)}\right)^{3}+24\left(\mu^{(2)}\right)^{2} \pi \alpha\left(c^{(2)}\right)^{5 / 2}\left(\mu^{(1)}\right)^{2}+
$$$$
4 \pi \mu^{(2)} \alpha\left(c^{(2)}\right)^{11 / 2}\left(\mu^{(1)}\right)^{3}-12 \pi\left(\mu^{(2)}\right)^{2} \alpha\left(c^{(2)}\right)^{11 / 2}\left(\mu^{(1)}\right)^{2}+12 \pi\left(\mu^{(2)}\right)^{3} \alpha\left(c^{(2)}\right)^{11 / 2} \mu^{(1)}+
$$$$
16\left(\mu^{(2)}\right)^{3} \pi^{2} \alpha^{3}\left(c^{(2)}\right)^{7 / 2} \mu^{(1)}-24\left(\mu^{(2)}\right)^{3} \pi \alpha\left(c^{(2)}\right)^{7 / 2} \mu^{(1)}+24 \mu^{(2)} \pi \alpha\left(c^{(2)}\right)^{7 / 2}\left(\mu^{(1)}\right)^{3}+
$$$$
8\left(c^{(2)}\right)^{5} \pi^{1 / 2} \mu^{(1)}\left(\mu^{(2)}\right)^{3}+6\left(c^{(2)}\right)^{6} \pi^{1 / 2}\left(\mu^{(1)} \mu^{(2)}\right)^{2}+4\left(c^{(2)}\right)^{3} \pi^{1 / 2}\left(\mu^{(1)}\right)^{4}+
$$$$
6\left(c^{(2)}\right)\left(\mu^{(2)}\right)^{4} \alpha^{2} \pi^{3 / 2}-12\left(c^{(2)}\right)^{3}\left(\mu^{(2)}\right)^{4} \alpha^{2} \pi^{3 / 2}+6\left(c^{(2)}\right)^{5}\left(\mu^{(2)}\right)^{4} \alpha^{2} \pi^{3 / 2}+
$$$$
8\left(\mu^{(2)}\right)^{4} \pi^{2} \alpha^{3}\left(c^{(2)}\right)^{3 / 2}+4\left(\mu^{(2)}\right)^{4} \pi \alpha\left(c^{(2)}\right)^{3 / 2}-4\left(\mu^{(2)}\right)^{4} \pi^{2} \alpha^{3}\left(c^{(2)}\right)^{9 / 2}+
$$$$
8\left(\mu^{(2)}\right)^{4} \pi \alpha\left(c^{(2)}\right)^{9 / 2}+4 \alpha^{4}\left(\mu^{(2)}\right)^{4} \pi^{5 / 2} c^{(2)}+6 \alpha^{4}\left(\mu^{(2)}\right)^{4} \pi^{5 / 2}\left(c^{(2)}\right)^{2}+
$$$$
4 \alpha^{4}\left(\mu^{(2)}\right)^{4} \pi^{5 / 2}\left(c^{(2)}\right)^{3}+\alpha^{4}\left(\mu^{(2)}\right)^{4} \pi^{5 / 2}\left(c^{(2)}\right)^{4}-8\left(\mu^{(2)}\right)^{4} \pi \alpha\left(c^{(2)}\right)^{5 / 2}-
$$$$
4 \pi\left(\mu^{(2)}\right)^{4} \alpha\left(c^{(2)}\right)^{11 / 2}-8\left(\mu^{(2)}\right)^{4} \pi^{2} \alpha^{3}\left(c^{(2)}\right)^{7 / 2}+4\left(c^{(2)}\right)^{2} \pi^{1 / 2}\left(\mu^{(2)}\right)^{3} \mu^{(1)}+
$$$$
4\left(c^{(2)}\right)^{2} \pi^{1 / 2} \mu^{(2)}\left(\mu^{(1)}\right)^{3}-8\left(c^{(2)}\right)^{3} \pi^{1 / 2} \mu^{(1)}\left(\mu^{(2)}\right)^{3}+4 \pi^{2}\left(\mu^{(2)}\right)^{4} \alpha^{3}\left(c^{(2)}\right)^{1 / 2}+
$$$$
\left.4 \pi^{2}\left(\mu^{(2)}\right)^{3} \alpha^{3}\left(c^{(2)}\right)^{1 / 2} \mu^{(1)}\right] .
$$ 


\section{References}

Abramowitz M., Stegun I.A. (Eds.), Handbook of Mathematical Functions, with Formulas, Graphs, and Mathematical Tables, Dover, New York, 1965.

Andrianov I.V., Bolshakov V.I., Danishevs'kyy V.V., Weichert D., 2007. Asymptotic simulation of imperfect bonding in periodic fibre-reinforced composite materials under axial shear. Int. J. Mech. Sci. 49, 1344-1354.

Andrianov I.V., Bolshakov V.I., Danishevs'kyy V.V., Weichert D., 2008. Higherorder asymptotic homogenization and wave propagation in periodic composite materials. Proc. R. Soc. A 464, 1181-1201.

Andrianov I.V., Bolshakov V.I., Danishevs'kyy V.V., Weichert D., 2010. Asymptotic study of imperfect interfaces in conduction through a granular composite material. Proc. R. Soc. A 466, 2707-2725.

Andrianov I.V., Danishevs'kyy V.V., Ryzhkov O.I., Weichert D., 2013. Dynamic homogenization and wave propagation in a nonlinear 1D composite material, Wave Motion 50, 271-281.

Andrianov I.V., Danishevs'kyy V.V., Ryzhkov O.I., Weichert D., 2014. Numerical study of formation of solitary strain waves in a nonlinear elastic layered composite material. Wave Motion 51, 405-417.

Andrianov I.V., Danishevs'kyy V.V., Topol H., Weichert D., 2011. Homogenization of a 1D nonlinear dynamical problem for periodic composites. ZAMM 91, $523-534$.

Auriault J.-L., Boutin C., 2012. Long wavelength inner-resonance cut-off frequencies in elastic composite materials. Int. J. Solids Struct. 49, 3269-3281. Bakhvalov N.S., Panasenko G.P., 1989. Homogenization: Averaging Processes in Periodic Media. Mathematical Problems in Mechanics of Composite Materials. Dordrecht, Kluwer.

Bensoussan A., Lions J.-L., Papanicolaou G., 1978. Asymptotic analysis for periodic structures. Amsterdam, North-Holland. 
Benveniste Y., Miloh T., 2001. Imperfect soft and stiff interfaces in twodimensional elasticity. Mech. Mater. 33, 309-324.

Berezovski A., Engelbrecht J., Berezovski M., 2011a. Waves in microstructured solids: a unified viewpoint of modeling. Acta Mech. 220, 349-363.

Berezovski A., Engelbrecht J., Maugin G.A., 2011b. Generalized thermomechanics with internal variables. Arch. Appl. Mech. 81, 229-240.

Bloch F., 1928. Über die Quantenmechanik der Elektronen in Kristallgittern. Z. Physik 52, 555-600.

Boutin C., Auriault J.L., 1993. Rayleigh scattering in elastic composite materials, Int. J. Eng. Sci. 31, 1669-1689.

Brillouin L., 2003. Wave Propagation in Periodic Structures: Electric Filters and Crystal Lattices, 2nd edn. Mineola, New York, Dover.

Chen W., Fish J, 2001. A dispersive model for wave propagation in periodic heterogeneous media based on homogenization with multiple spatial and temporal scales. J. Appl. Mech. 68, 153-161.

Cherednichenko K.D., Smyshlyaev V.P., Zhikov V.V., 2006. Non-local homogenized limits for composite media with highly anisotropic periodic fibres. Proc. R. Soc. Edinburgh 136A, 87-114.

Christensen R.M., 2005. Mechanics of Composite Materials, 2nd ed. Mineola, New York, Dover.

Christov C.I., Maugin G.A., Velarde M.E., 1996. Well-posed Boussinesq paradigm with purely spatial higher-order derivatives. Phys. Rev. E 54, 3621-3638.

Cosserat E., Cosserat F., 1909. Théorie des corps déformables. Paris, Hermann et fils.

Craster R.V., Joseph L.M., Kaplunov J., 2014. Long-wave asymptotic theories: The connection between functionally graded waveguides and periodic media. Wave Motion 51, 581-588. 
Craster R.V., Kaplunov J., Nolde E., Guenneau S., 2012. Bloch dispersion and high frequency homogenization for separable doubly-periodic structures. Wave Motion 49, 333-346.

Craster R.V., Kaplunov J., Pichugin A.V., 2010a. High frequency homogenization for periodic media. Proc. R. Soc. Lond. A 466, 2341-2362.

Craster R.V., Kaplunov J., Postnova J., 2010b. High frequency asymptotics homogenization and localization for lattices. Q. J. Mech. Appl. Math. 63, 497519.

Drazin P.G., Johnson R.S., 1989. Solitons: an Introduction. Cambridge, New York, Cambridge University Press.

Engelbrecht J., Berezovski A., Salupere A., 2007. Nonlinear deformation waves in solids and dispersion. Wave Motion 44, 493-500.

Engelbrecht J., Salupere A., Tamm K., 2011. Waves in microstructured solids and the Boussinesq paradigm, Wave Motion 48, 717-726.

Erofeev V.I., 2003. Wave Processes in Solids with Microstructure. Singapore, World Scientific.

Espinosa H.D., Dwivedi S.K., Lu H-C., 2000. Modelling impact induced delamination of woven fibre reinforced composites with contact/cohesive laws. Comput. Methods Appl. M. 183, 259-290.

Espinosa H.D., Zavattieri P.D., Dwivedi S.K., 1998. A finite deformation continuum/discrete model for the description of fragmentation and damage in brittle materials. J. Mech. Phys. Solids 46, 1909-1942.

Fish J., Chen W., 2004. Space-time multiscale model for wave propagation in heterogeneous media. Comput. Methods Appl. Math. 193, 4837-4856.

Goland M., Reissner E., 1944. The stresses in cemented joints. J. Appl. Mech. 11, A17-A27.

Goldenveizer A.L., Kaplunov J.D., Nolde E.V., 1993. On Timoshenko-Reissner type theories of plates and shells. Int. J. Solids Struct. 30, 675-694. 
Hashin Z., 1965. On elastic behaviour of fibre reinforced materials of arbitrary transverse phase geometry. J. Mech. Phys. Solids 13, 119-134.

Hashin Z., 1992. Extremum principles for elastic heterogeneous media with imperfect interface and their application to bounding of effective elastic moduli. J. Mech. Phys. Solids 40, 767-781.

Herrmann G., Achenbach J.D., 1968. Application of theories of generalized Cosserat continua to the dynamics of composite materials. In: E. Kröner (Ed.) Mechanics of Generalized Continua. Berlin, Heidelberg, New York, Springer, 69-79.

Jeffrey A., Engelbrecht J., 1994. Nonlinear Waves in Solids. Wien, Springer.

Kalamkarov A.L., Andrianov I.V., Danishevs'kyy V.V., 2009. Asymptotic homogenization of composite materials and structures, Appl. Mech. Rev. 62, 030802.

Kaplunov J.D., Kossovich L.Yu., Nolde E.V., 1998. Dynamics of thin walled elastic bodies. San Diego, Calif, Academic Press.

Kaplunov J.D., Nolde E.V., Rogerson G.A., 2000. A low-frequency model for dynamic motion in pre-stressed incompressible elastic structures. Proc. R. Soc. A 456, 2589-2610.

Kunin I.A., 1982. Elastic Media with Microstructure. Vol. 1. One-Dimensional Models. Berlin, New York, Springer.

Kushwaha M.S., Halevi P., Martinez G., Dobrzynski L., Djafari-Rouhani B., 1994. Theory of acoustic band structure of periodic elastic composites. Phys. Rev. B 49, 2313-2322.

Le Roux J., 1911. Étude géométrique de la torsion et de la flexion dans la déformation. Ann. Scient. de l'École Normale Sup, 3e série, tome 28, 523579.

Levy A.J., 2000. The fiber composite with nonlinear interface. Part I: Axial tension. J. Appl. Mech. 67, 727-732.

Levy A.J., Dong Z., 1998. Effective transverse response of fiber composites with 
nonlinear interface. J. Mech. Phys. Solids 46, 1279-1300.

Lipton R., Vernescu B., 1995. Variational methods, size effects and extremal microgeometries for elastic composites with imperfect interface. Math. Mod. Meth. Appl. S. 5, 1139-1173.

Lur'e A.I., 1990. Nonlinear Theory of Elasticity. Amsterdam, North-Holland.

Lutianov M., Rogerson G.A., 2010. Long wave motion in layered elastic media. Int. J. Eng. Sci. 48, 1856-1871.

Mal A.K., Bose S.K., 1975. Dynamic elastic moduli of a suspension of imperfectly bonded spheres. Proc. Cambridge Philos. Soc. 76, 587-600.

Maugin G.A., 1999. Nonlinear Waves in Elastic Crystals. Oxford, Oxford University Press.

McPhedran R.C., Movchan A.B., Movchan N.V., 2009. Platonic crystals: Bloch bands, neutrality and defects. Mech. Mater. 41, 356-363.

Mindlin R.D., 1964. Microstructure in linear elasticity. Arch. Ration. Mech. Anal. $16,51-78$.

Movchan A.B., Movchan N.V., Haq S., 2006. Localised vibration modes and stop bands for continuous and discrete periodic structures. Mater. Sci. Eng. 431, $175-183$.

Movchan A.B., Movchan N.V., McPhedran R.C., 2007. Bloch-Floquet bending waves in perforated thin plates. Proc. Roy. Soc. Lond. A 463, 2505-2518.

Movchan A.B., Movchan N.V., Poulton C.G., 2002. Asymptotic Models of Fields in Dilute and Densely Packed Composites. London, Imperial College Press.

Mukhomodyarov R.R., Rogerson G.A., 2012. Long-wave dispersion phenomena in a layer subject to elastically restrained boundary conditions. ZAMP 63, 171188.

Needleman A., 1990. An analysis of tensile decohesion along an interface. J. Mech. Phys. Solids 38, 289-324.

Needleman A., 1992. Micromechanical modelling of interfacial decohesion. Ultramicroscopy 40, 203-214. 
Nguyen Ch., Levy A.J., 2009. An exact theory of interfacial debonding in layered elastic composites. Int. J. Solids Struct. 46, 2712-2723.

Nolde E., Craster R.V., Kaplunov J., 2011. High frequency homogenization for structural mechanics. J. Mech. Phys. Solids 59, 651-671.

Parnell W.J., Abrahams I.D., 2006. Dynamic homogenization in periodic fibre reinforced media. Quasi-static limit for SH waves. Wave Motion 43, 474-498.

Perrins W.T., McKenzie D.R., McPhedran R.C., 1979. Transport properties of regular arrays of cylinders. Proc. R. Soc. Lond. A 369, 207-225.

Pichugin A.V., Askes H., Tyas A., 2008. Asymptotic equivalence of homogenisation procedures and fine-tuning of continuum theories. J. Sound Vib. 313, 858-874.

Polimeno U., Meo M., 2008. Understanding the effect of boundary conditions on damage identification process when using non-linear elastic wave spectroscopy methods. Int. J. Non-Linear Mech. 43, 187-193.

Porubov A.V., 2003. Amplification of Nonlinear Strain Waves in Solids. Singapore, World Scientific.

Porubov A.V., Aero E.L., Maugin G.A., 2009. Two approaches to study essentially nonlinear and dispersive properties of the internal structure of materials. Phys. Rev. E 79, 046608.

Porubov A.V., Pastrone F., 2004. Non-linear bell-shaped and kink-shaped strain waves in microstructural solids. Int. J. Non-Linear Mech. 39, 1289-1299.

Randrüüt M., Braun M., 2010. On one-dimensional solitary waves in microstructured solids. Wave Motion 47, 217-230.

Rogerson G.A., Kaplunov J.D., Nolde E.V., 2006. An asymptotic analysis of initial-value problems for thin elastic plates. Proc Royal Soc. A 462, 25412561.

Rogerson G.A., Prikazchikova L.A., 2009. Generalisations of long wave theories for pre-stressed compressible elastic plates. Int. J. Non-Linear Mech. 44, 520529. 
Rogerson G.A., Sandiford K.J., Prikazchikova L.A., 2007. Abnormal long wave dispersion phenomena in a slightly compressible elastic plate with non-classical boundary conditions. Int. J. Non-Linear Mech. 42, 298-309.

Samsonov A.M., 2001. Strain Solitons in Solids and How to Construct Them. Boca Raton, CRC Press.

Sanchez-Palencia E., 1980. Non-homogenous media and vibration theory. Berlin, New York, Springer.

Smyshlyaev V.P., 2009. Propagation and localization of elastic waves in highly anisotropic periodic composites via two-scale homogenization. Mech. Mater. $41,434-447$.

Soubestre J., Boutin C., 2012. Non-local dynamic behaviour of linear fibre reinforced materials. Mech. Mater. 55, 16-32.

Sun C.-T., Achenbach J.D., Herrmann G., 1968. Continuum theory for a laminated medium. Trans. ASME J. Appl. Mech. 35, 467-475.

Tan H., Liu C., Huang Y., Geubelle P.H., 2005. The cohesive law for the particle/matrix interfaces in high explosives. J. Mech. Phys. Solids 53, 18921917.

Tvergaard V., 1990. Effect of fibre debonding in a whisker-reinforced metal. Mater. Sci. Eng. A 125, 203-213.

Tvergaard V., 1995. Fiber debonding and breakage in a whisker reinforced metal. Mater. Sci. Eng. A 90, 215-222.

William T.D., Parnell W.J., 2014. Effective antiplane elastic properties of an orthotropic solid weakened by a periodic distribution of cracks. Quart. J. Mech. Applied Mathematics 67, 311-342.

Zaitsev V.Yu., Nazarov V.E., Talanov V.I., 2006. "Nonclassical" manifestations of microstructure-induced nonlinearities: new prospects for acoustic diagnostics. Phys.-Usp. 49, 89-94.

Zumpano, Meo M., 2008. Damage localization using transient non-linear elastic wave spectroscopy on composite structures. Int. J. Non-Linear Mech. 43, $217-$ 
230.

$-37-$ 